\title{
On the $k$-error linear complexity of binary sequences derived from polynomial quotients
}

\author{
Zhixiong Chen \\ School of Applied Mathematics, Putian University, \\ Putian, Fujian 351100, P. R. China \\ ptczx@126.com \\ Zhihua Niu \\ School of Computer Engineering and Science, Shanghai University, \\ Shangda Road, Shanghai 200444, P. R. China \\ zhniu@staff.shu.edu.cn \\ Chenhuang $\mathrm{Wu}$ \\ School of Applied Mathematics, Putian University, \\ Putian, Fujian 351100, P. R. China
}

August 14, 2021

\begin{abstract}
We investigate the $k$-error linear complexity of $p^{2}$-periodic binary sequences defined from the polynomial quotients (including the well-studied Fermat quotients), which is defined by

$$
q_{p, w}(u) \equiv \frac{u^{w}-u^{w p}}{p} \bmod p \text { with } 0 \leq q_{p, w}(u) \leq p-1, u \geq 0,
$$

where $p$ is an odd prime and $1 \leq w<p$. Indeed, first for all integers $k$, we determine exact values of the $k$-error linear complexity over the finite field $\mathbb{F}_{2}$ for these binary sequences under the assumption of 2 being a primitive root modulo $p^{2}$, and then we determine their $k$-error linear complexity over the finite field $\mathbb{F}_{p}$ for either $0 \leq k<p$ when $w=1$ or $0 \leq k<p-1$ when $2 \leq w<p$. Theoretical results obtained indicate that such sequences possess 'good' error linear complexity.
\end{abstract}

Keywords: Fermat quotients, Polynomial quotients, Binary sequences, Linear complexity, $k$-Error linear complexity, Cryptography

MSC(2010): 94A55, 94A60, 65C10 


\section{Introduction}

For an odd prime $p$ and integers $u \geq 0$ with $\operatorname{gcd}(u, p)=1$, the Fermat quotient $q_{p}(u)$ is defined as the unique integer

$$
q_{p}(u) \equiv \frac{u^{p-1}-1}{p} \bmod p \text { with } 0 \leq q_{p}(u) \leq p-1,
$$

and

$$
q_{p}(l p)=0, \quad l \in \mathbb{Z} .
$$

An equivalent definition of the Fermat quotient is given below

$$
q_{p}(u) \equiv \frac{u^{p-1}-u^{p(p-1)}}{p} \bmod p, u \geq 0 .
$$

For any fixed positive integer $w$, by the fact that

$$
\left(u^{w}\right)^{p} \equiv u^{w} \bmod p, u \geq 0
$$

from the Fermat Little Theorem, Chen and Winterhof extended (1) to define

$$
q_{p, w}(u) \equiv \frac{u^{w}-u^{w p}}{p} \bmod p \text { with } 0 \leq q_{p, w}(u) \leq p-1, u \geq 0,
$$

which is called a polynomial quotient in [14]. In fact $q_{p, p-1}(u)=q_{p}(u)$. It is easy to see that

$$
q_{p, w}(u+l p)=q_{p, w}(u)+w l u^{w-1}(\bmod p)
$$

if $\operatorname{gcd}(u, p)=1$, and

$$
q_{p, w}(l p)=\left\{\begin{array}{ll}
0, & \text { if } w>1, \\
l, & \text { if } w=1,
\end{array} \quad l=0, \ldots, p-1\right.
$$

Many number theoretic and cryptographic questions as well as measures of pseudorandomness have been studied for Fermat quotients and their generalizations [1, 5, 7, 9, 10, 11, 13, 14, 15, 16, 25, 26, 29, 31, 37, 38, 40, 41, 42, 43, 44, 45.

In this paper, we still concentrate on certain binary sequences defined from the polynomial quotients (of course including the Fermat quotients) in the references. The first one is the binary threshold sequence $\left(e_{u}\right)$ studied in [10, 11, 12, 13, 17, 26] by defining

$$
e_{u}=\left\{\begin{array}{ll}
0, & \text { if } 0 \leq q_{p, w}(u) / p<\frac{1}{2}, \\
1, & \text { if } \frac{1}{2} \leq q_{p, w}(u) / p<1,
\end{array} \quad u \geq 0 .\right.
$$

The second one, by combining $q_{p, w}(u)$ with the Legendre symbol $(\dot{\bar{p}})$, is defined in [12, 17, 26, 31] by

$$
f_{u}= \begin{cases}0, & \text { if }\left(\frac{q_{p, w}(u)}{p}\right)=1 \text { or } q_{p, w}(u)=0, \quad u \geq 0 . \\ 1, & \text { otherwise, }\end{cases}
$$


(In fact, in [26, 31] $\chi$, a fixed multiplicative character modulo $p$ of order $m>1$, is applied to defining $m$-ary sequences $\left(\widetilde{f}_{u}\right)$ of discrete logarithms modulo a divisor $m$ of $p-1$ by

$$
\exp \left(2 \pi i \widetilde{f}_{u} / m\right)=\chi\left(q_{p, w}(u)\right), 0 \leq \widetilde{f}_{u}<m \quad \text { if } q_{p, w}(u) \not \equiv 0 \bmod p
$$

and $\widetilde{f}_{u}=0$ otherwise. When $m=2$, we have $\widetilde{f}_{u}=f_{u}$ for all $u \geq 0$.) We note that both $\left(e_{u}\right)$ and $\left(f_{u}\right)$ are $p^{2}$-periodic by (3).

The authors of [13, 31] investigated measures of pseudorandomness as well as linear complexity profile of $\left(e_{u}\right)$ and $\left(\tilde{f}_{u}\right)$ (of course including $\left.\left(f_{u}\right)\right)$ via certain character sums over Fermat quotients. The authors of [12, 26] determined the linear complexity (see below for the definition) of $\left(e_{u}\right)$ and $\left(f_{u}\right)$ if 2 is a primitive element modulo $p^{2}$, and later the authors of [10, 11, 17] extended to a more general setting of $2^{p-1} \not \equiv 1\left(\bmod p^{2}\right)$ when $w \in\{p-1,(p-1) / 2\}$. The authors of [17] also determined the trace representations of $\left(e_{u}\right)$ and $\left(f_{u}\right)$. In this paper, our main aim is to study the $k$-error linear complexity (see below for the definition) for $\left(e_{u}\right)$ and $\left(f_{u}\right)$. All results indicate that such sequences have desirable cryptographic features.

For our purpose, we need to describe $\left(e_{u}\right)$ and $\left(f_{u}\right)$ in an equivalent way. From (3), $q_{p, w}(-)$ induces a surjective map from $\mathbb{Z}_{p^{2}}^{*}$ (the group of invertible elements modulo $p^{2}$ ) to $\mathbb{Z}_{p}$ (the additive group of numbers modulo $p$ ). For each fixed $1 \leq w<p$, we define

$$
D_{l}=\left\{u: 0 \leq u<p^{2}, \operatorname{gcd}(u, p)=1, q_{p, w}(u)=l\right\}
$$

for $l=0,1, \ldots, p-1$. Each $D_{l}$ has the cardinality $\left|D_{l}\right|=p-1$ by (3). Here and hereafter, we use $|S|$ to denote the cardinality of a set $S$. Let $P=\{l p: 0 \leq l<p\}$, for $w \geq 2$ one can define $\left(e_{u}\right)$ and $\left(f_{u}\right)$ equivalently by

$$
e_{u}= \begin{cases}0, & \text { if } u \bmod p^{2} \in D_{0} \cup \cdots \cup D_{(p-1) / 2} \cup P, \\ 1, & \text { if } u \bmod p^{2} \in D_{(p+1) / 2} \cup \cdots \cup D_{p-1},\end{cases}
$$

and

$$
f_{u}= \begin{cases}0, & \text { if } u \bmod p^{2} \in \cup_{l \in Q} D_{l} \cup D_{0} \cup P, \\ 1, & \text { if } u \bmod p^{2} \in \cup_{l \in N} D_{l},\end{cases}
$$

respectively, where $Q$ is the set of quadratic residues modulo $p$ and $N$ is the set of quadratic non-residues modulo $p$. For $w=1$, it is easy to define $\left(e_{u}\right)$ and $\left(f_{u}\right)$ similarly by only redividing the set $P$.

We need to mention that, the following relation holds between $q_{p, w}(u)$ and $q_{p}(u)$ :

$$
q_{p, w}(u) \equiv-u^{w} w q_{p}(u) \bmod p
$$

for all $u \geq 0$ with $\operatorname{gcd}(u, p)=1$. If $w=l p$ for any positive integer $l$, we have $q_{p, l p}(u)=0$ by (7) and (4) for all $u \geq 0$. For any positive $w$ with $p \nmid w$, write $w=w_{1}+w_{2}(p-1)$ with $1 \leq w_{1} \leq p-1$ and $w_{2} \geq 0$, by (7) again one can get

$$
q_{p, w_{1}+w_{2}(p-1)}(u) \equiv-u^{w_{1}}\left(w_{1}-w_{2}\right) q_{p}(u) \equiv w_{1}^{-1}\left(w_{1}-w_{2}\right) q_{p, w_{1}}(u) \bmod p .
$$

Note that $w_{1} \not \equiv w_{2} \bmod p$ since $p \nmid w$. Hence, a polynomial quotient $q_{p, w}(-)$ with large $w$ can be reduced to the one with $1 \leq w_{1} \leq p-1$ and we restrict ourselves to $1 \leq w \leq p-1$ from now on. 
We conclude this section by introducing the notions of the linear complexity and the $k$-error linear complexity of periodic sequences.

Let $\mathbb{F}$ be a field. For a $T$-periodic sequence $\left(s_{u}\right)$ over $\mathbb{F}$, we recall that the linear complexity over $\mathbb{F}$, denoted by $L C^{\mathbb{F}}\left(\left(s_{u}\right)\right)$, is the least order $L$ of a linear recurrence relation over $\mathbb{F}$

$$
s_{u+L}=c_{L-1} s_{u+L-1}+\cdots+c_{1} s_{u+1}+c_{0} s_{u} \text { for } u \geq 0,
$$

which is satisfied by $\left(s_{u}\right)$ and where $c_{0} \neq 0, c_{1}, \ldots, c_{L-1} \in \mathbb{F}$. Let

$$
S(X)=s_{0}+s_{1} X+s_{2} X^{2}+\cdots+s_{T-1} X^{T-1} \in \mathbb{F}[X],
$$

which is called the generating polynomial of $\left(s_{u}\right)$. Then the linear complexity over $\mathbb{F}$ of $\left(s_{u}\right)$ is computed by

$$
L C^{\mathbb{F}}\left(\left(s_{u}\right)\right)=T-\operatorname{deg}\left(\operatorname{gcd}\left(X^{T}-1, S(X)\right)\right),
$$

see, e.g. [34 for details. For integers $k \geq 0$, the $k$-error linear complexity over $\mathbb{F}$ of $\left(s_{u}\right)$, denoted by $L C_{k}^{\mathbb{F}}\left(\left(s_{u}\right)\right)$, is the smallest linear complexity (over $\mathbb{F}$ ) that can be obtained by changing at most $k$ terms of the sequence per period, see [46, 36], and see [21] for the related even earlier defined sphere complexity. Clearly $L C_{0}^{\mathbb{F}}\left(\left(s_{u}\right)\right)=L C^{\mathbb{F}}\left(\left(s_{u}\right)\right)$ and

$$
T \geq L C_{0}^{\mathbb{F}}\left(\left(s_{u}\right)\right) \geq L C_{1}^{\mathbb{F}}\left(\left(s_{u}\right)\right) \geq \ldots \geq L C_{k}^{\mathbb{F}}\left(\left(s_{u}\right)\right)=0
$$

when $k$ equals the number of nonzero terms of $\left(s_{u}\right)$ per period, i.e., the weight of $\left(s_{u}\right)$.

The linear complexity and the $k$-error linear complexity are important cryptographic characteristics of sequences and provide information on the predictability and thus unsuitability for cryptography. For a sequence to be cryptographically strong, its linear complexity should be large, but not significantly reduced by changing a few terms. And according to the Berlekamp-Massey algorithm [35], the linear complexity should be at least a half of the period.

Instead of studying $\left(e_{u}\right)$ and $\left(f_{u}\right)$ directly, we define the $p^{2}$-periodic binary sequence $\left(h_{u}\right)$ by

$$
h_{u}= \begin{cases}1, & \text { if } u \bmod p^{2} \in \cup_{l \in \mathcal{I}} D_{l}, \quad u \geq 0, \\ 0, & \text { otherwise }\end{cases}
$$

for $w \geq 2$, and

$$
h_{u}= \begin{cases}1, & \text { if } u \bmod p^{2} \in \cup_{l \in \mathcal{I}}\left(D_{l} \cup\{l p\}\right), \quad u \geq 0, \\ 0, & \text { otherwise }\end{cases}
$$

for $w=1$, where $\mathcal{I}$ is a non-empty subset of $\{0,1, \ldots, p-1\}$, and investigate the $k$-error linear complexity over $\mathbb{F}_{2}$ for $\left(h_{u}\right)$ in Section 2 . In Section 3 , we investigate the $k$-error linear complexity over $\mathbb{F}_{p}$ for $\left(h_{u}\right)$. Although $\left(h_{u}\right)$ is a binary sequence, it is constructed based on the polynomial quotients modulo $p$ (note that the linear complexity over $\mathbb{F}_{p}$ of the polynomial quotients is $p+w$, see a proof in [37] for the Fermat quotients), thus, it is natural to consider the $k$-error linear complexity over $\mathbb{F}_{p}$ for $\left(h_{u}\right)$. In fact, it is also motivated by the ideas of [3, 4] and partially [2, 5, 8, 18, 28, 30, 32, 33. 


\section{$2 \quad k$-Error Linear Complexity over $\mathbb{F}_{2}$}

First we present some auxiliary statements. Define

$$
D_{l}(X)=\sum_{u \in D_{l}} X^{u} \in \mathbb{F}_{2}[X]
$$

for $0 \leq l<p$.

Lemma 1. Let $\theta \in \overline{\mathbb{F}}_{2}$ be a primitive $p$-th root of unity. For $0 \leq l<p$, we have

$$
D_{l}\left(\theta^{m}\right)= \begin{cases}0, & \text { if } m \equiv 0 \quad(\bmod p), \\ 1, & \text { otherwise. }\end{cases}
$$

Proof. For any fixed $1 \leq v<p$, the numbers $v+m p$ belong to different $D_{l}(0 \leq l<p)$ when $m$ runs through the set $\{0,1, \ldots, p-1\}$ by (3), hence we have

$$
\left\{u \quad(\bmod p): u \in D_{l}\right\}=\mathbb{Z}_{p}^{*}, 0 \leq l<p .
$$

We note that in the definition of $D_{l}$, we restrict $1 \leq w<p$. For $0 \leq l<p$, we derive

$$
D_{l}\left(\theta^{m}\right)=\sum_{u \in D_{l}} \theta^{m u}=\sum_{j \in \mathbb{Z}_{p}^{*}} \theta^{m j},
$$

which deduces the desired result for different $m$ modulo $p$. The calculations here are performed in finite fields with characteristic two.

Lemma 2. Let $\theta \in \overline{\mathbb{F}}_{2}$ be a primitive $p$-th root of unity and $G(X) \in \mathbb{F}_{2}[X]$ with $1 \leq$ $\operatorname{deg}(G(X))<p$. If 2 is a primitive root modulo $p$, we have

$$
G(\theta)=1 \Longleftrightarrow G(X)=X+X^{2}+\ldots+X^{p-1},
$$

or

$$
G(\theta)=0 \Longleftrightarrow G(X)=1+X+X^{2}+\ldots+X^{p-1} .
$$

Proof. We only show the first assertion. Since 2 is a primitive root modulo $p$, we see that $1+X+X^{2}+\ldots+X^{p-1}$ is the minimal irreducible polynomial with the root $\theta$. So if $G(\theta)=1$, we derive

$$
\left(1+X+X^{2}+\ldots+X^{p-1}\right) \mid(G(X)-1) .
$$

With the restriction on $\operatorname{deg}(G(X))$, we get $G(X)=X+X^{2}+\ldots+X^{p-1}$. The converse is true after simple calculations.

Now we present our main results.

Theorem 1. Let $\left(h_{u}\right)$ be the binary sequence of period $p^{2}$ defined in (9) using polynomial quotients (2) with $2 \leq w \leq p-1$ and a non-empty subset $\mathcal{I}$ of $\{0,1, \ldots, p-1\}$ with $1 \leq|\mathcal{I}| \leq(p-1) / 2$. If 2 is a primitive root modulo $p^{2}$, then the $k$-error linear complexity over $\mathbb{F}_{2}$ of $\left(h_{u}\right)$ satisfies

$$
L C_{k}^{\mathbb{F}_{2}}\left(\left(h_{u}\right)\right)=\left\{\begin{array}{cl}
p^{2}-1, & \text { if } k=0 \\
p^{2}-p+1, & \text { if } 1 \leq k<p-1 \\
p^{2}-p, & \text { if } p-1 \leq k<(p-1)|\mathcal{I}|,|\mathcal{I}|>1, \\
0, & \text { if } k \geq(p-1)|\mathcal{I}|
\end{array}\right.
$$


if $|\mathcal{I}|$ is odd, and otherwise

$$
L C_{k}^{\mathbb{F}_{2}}\left(\left(h_{u}\right)\right)=\left\{\begin{array}{cl}
p^{2}-p, & \text { if } 0 \leq k<(p-1)|\mathcal{I}| \\
0, & \text { if } k \geq(p-1)|\mathcal{I}|
\end{array}\right.
$$

Proof. From the construction of $\left(h_{u}\right)$, there are $(p-1)|\mathcal{I}|$ many 1's in one period of $\left(h_{u}\right)$ since each $D_{l}$ contains $p-1$ many elements. Changing all terms of 1's will lead to the zero sequence. So we always assume that $k<(p-1)|\mathcal{I}|$. Let

$$
H_{k}(X)=\sum_{l \in \mathcal{I}} D_{l}(X)+e(X) \in \mathbb{F}_{2}[X]
$$

be the generating polynomial of the sequence obtained from $\left(h_{u}\right)$ by changing exactly $k$ terms of $\left(h_{u}\right)$ per period, where $e(X)$ is the corresponding error polynomial with $k$ many monomials. We note that $H_{k}(X)$ is a nonzero polynomial due to $k<(p-1)|\mathcal{I}|$. We will consider the common roots of $H_{k}(X)$ and $X^{p^{2}}-1$, i.e., the roots of the form $\beta^{n}\left(n \in \mathbb{Z}_{p^{2}}\right)$ for $H_{k}(X)$, where $\beta \in \overline{\mathbb{F}}_{2}$ is a primitive $p^{2}$-th root of unity. The number of the common roots will help us to derive the values of $k$-error linear complexity of $\left(h_{u}\right)$ by (8).

On the one hand, we assume that $H_{k}\left(\beta^{n_{0}}\right)=0$ for some $n_{0} \in \mathbb{Z}_{p^{2}}^{*}$. Since 2 is a primitive root modulo $p^{2}$, for each $n \in \mathbb{Z}_{p^{2}}^{*}$, there exists a $0 \leq j_{n}<(p-1) p$ such that $n \equiv n_{0} 2^{j_{n}} \bmod p^{2}$. Then we have

$$
H_{k}\left(\beta^{n}\right)=H_{k}\left(\beta^{n_{0} 2^{j n}}\right)=H_{k}\left(\beta^{n_{0}}\right)^{2^{j n}}=0,
$$

that is, all ( $p^{2}-p$ many) elements $\beta^{n}$ for $n \in \mathbb{Z}_{p^{2}}^{*}$ are roots of $H_{k}(X)$. Hence we have

$$
\Phi(X) \mid H_{k}(X) \text { in } \overline{\mathbb{F}}_{2}[X]
$$

where

$$
\Phi(X)=1+X^{p}+X^{2 p}+\ldots+X^{(p-1) p} \in \mathbb{F}_{2}[X],
$$

the roots of which are exactly $\beta^{n}$ for $n \in \mathbb{Z}_{p^{2}}^{*}$. Let

$$
H_{k}(X) \equiv \Phi(X) \pi(X) \quad\left(\bmod X^{p^{2}}-1\right) .
$$

Using the fact that

$$
X^{p} \Phi(X) \equiv \Phi(X) \quad\left(\bmod X^{p^{2}}-1\right),
$$

we restrict $\operatorname{deg}(\pi(X))<p$ and write

$$
\pi(X)=X^{v_{0}}+X^{v_{1}}+\ldots+X^{v_{t-1}} \text { with } 0 \leq v_{0}<v_{1}<\ldots<v_{t-1}<p,
$$

where $t \geq 1$ since $H_{k}(X)$ is a nonzero polynomial. Then the exponent of each monomial in $\Phi(X) \pi(X) \bmod X^{p^{2}}-1$ forms the set

$$
\left\{v_{j}+l p: 0 \leq j \leq t-1,0 \leq l \leq p-1\right\},
$$

which can be divided into two sets $A$ and $B$ with

$$
A=\left\{v_{j}+l p: 0 \leq j \leq t-1,0 \leq l \leq p-1, v_{j} \neq 0, q_{p, w}\left(v_{j}+l p\right) \in \mathcal{I}\right\},
$$




$$
B=\left\{v_{j}+l p: 0 \leq j \leq t-1,0 \leq l \leq p-1\right\} \backslash A .
$$

We note that by (3) $A$ contains $|A|$ many numbers with

$$
|A|=\left\{\begin{array}{cl}
(t-1)|\mathcal{I}|, & \text { if } v_{0}=0 \\
t|\mathcal{I}|, & \text { otherwise }
\end{array}\right.
$$

and $B$ contains $t p-|A|$ many numbers.

Hence, from (11) and (12), we find that the set of the exponents of monomials in $e(X)$ is

$$
\left(\cup_{l \in \mathcal{I}} D_{l} \backslash A\right) \cup B
$$

the cardinality of which is

$$
(p-1)|\mathcal{I}|-|A|+|B|=(p-1)|\mathcal{I}|+t p-\left\{\begin{array}{cl}
2(t-1)|\mathcal{I}|, & \text { if } v_{0}=0 \\
2 t|\mathcal{I}|, & \text { otherwise }
\end{array}\right.
$$

That is, $k=(p-1)|\mathcal{I}|-|A|+|B|$ since $e(X)$ contains $k$ many terms. However, the assumption of $1 \leq|\mathcal{I}| \leq(p-1) / 2$ implies $t p-2 t|\mathcal{I}|>0$ and hence

$$
(p-1)|\mathcal{I}|-|A|+|B|>(p-1)|\mathcal{I}|>k,
$$

a contradiction. So $H_{k}\left(\beta^{n}\right) \neq 0$ for all $n \in \mathbb{Z}_{p^{2}}^{*}$.

On the other hand, by Lemma 1 we get

$$
H_{k}\left(\beta^{i p}\right)=e\left(\beta^{i p}\right)+ \begin{cases}0, & \text { if } i=0 \\ |\mathcal{I}|, & \text { if } 1 \leq i<p .\end{cases}
$$

Hence below we only need to consider the number of roots of the form $\beta^{i p}(0 \leq i<p)$ for $H_{k}(X)$.

First, if $|\mathcal{I}|$ is odd, for $k=0$ (in this case $e(X)$ will not occur) it is easy to see that

$$
L C_{0}^{\mathbb{F}_{2}}\left(\left(h_{u}\right)\right)=L C^{\mathbb{F}_{2}}\left(\left(h_{u}\right)\right)=p^{2}-1 .
$$

For $1 \leq k<p-1$, we first consider $e(X)=X^{i_{0} p}$ for some $0 \leq i_{0}<p$, i.e., $\left(h_{u}\right)$ is changed only one term at the position $i_{0} p$ per period, we have $e\left(\beta^{i p}\right)=1$ for all $0 \leq i<p$ and there are exactly $p-1$ many $\beta^{i p}(1 \leq i<p)$ such that $H_{k}\left(\beta^{i p}\right)=0$. However, for any other $e(X)$ with $k(1 \leq k<p-1)$ terms, the number of such kind of roots of $H_{k}(X)$ does not increase, since $e(X)$ satisfying

$$
e\left(\beta^{i p}\right)= \begin{cases}0, & \text { if } i=0, \\ 1, & \text { if } 1 \leq i<p,\end{cases}
$$

which guarantees all $\beta^{i p}(0 \leq i<p)$ are roots of $H_{k}(X)$, should be of the form

$$
e(X) \equiv X+X^{2}+\ldots+X^{p-1} \quad\left(\bmod X^{p}-1\right)
$$

by Lemma 2 , in other words, $e(X)$ should contain at least $p-1$ many terms if the number of roots of $H_{k}(X)$ increases (from $p-1$ to $p$ ). So we derive

$$
L C_{k}^{\mathbb{F}_{2}}\left(\left(h_{u}\right)\right)=L C_{1}^{\mathbb{F}_{2}}\left(\left(h_{u}\right)\right)=p^{2}-p+1 \text { for } 1 \leq k<p-1 .
$$


For $p-1 \leq k<(p-1)|\mathcal{I}|$ and $|\mathcal{I}|>1$, one can choose $e(X)$ with $p-1$ terms, as mentioned above, of the form $e(X) \equiv X+X^{2}+\ldots+X^{p-1} \bmod X^{p}-1$ such that all $\beta^{i p}$ 's $(0 \leq i<p)$ are roots of $H_{k}(X)$. Hence, we get

$$
L C_{k}^{\mathbb{F}_{2}}\left(\left(h_{u}\right)\right)=L C_{p-1}^{\mathbb{F}_{2}}\left(\left(h_{u}\right)\right)=p^{2}-p \text { for } p-1 \leq k<(p-1)|\mathcal{I}| .
$$

Second, we turn to the case of even $|\mathcal{I}|$. When $k=0$, all $\beta^{i p}(0 \leq i<p)$ are roots of $H_{k}(X)$ from 13 . No other possible roots of the form $\beta^{n}$ will occur for $1 \leq k<(p-1)|\mathcal{I}|$. So we have

$$
L C_{k}^{\mathbb{F}_{2}}\left(\left(h_{u}\right)\right)=L C_{0}^{\mathbb{F}_{2}}\left(\left(h_{u}\right)\right)=p^{2}-p \text { for } 0 \leq k<(p-1)|\mathcal{I}| .
$$

We complete the proof.

In Theorem 1, we restrict $1 \leq|\mathcal{I}| \leq(p-1) / 2$. For $|\mathcal{I}|>(p-1) / 2$, we can similarly consider the complementary sequence, denoted by $\left(h_{u}^{\prime}\right)$, of $\left(h_{u}\right)$, i.e., $h_{u}^{\prime} \equiv h_{u}+1 \bmod 2$ for all $u \geq 0$. The difference between the (k-error) linear complexity of $\left(h_{u}\right)$ and that of $\left(h_{u}^{\prime}\right)$ is at most 1 by the fact that

$$
\frac{H^{c}(X)+e(X)}{X^{p^{2}}-1}=\frac{H(X)+e(X)}{X^{p^{2}}-1}+\frac{1}{X-1},
$$

where $H^{c}(X)$ is the generating polynomial of $\left(h_{u}^{\prime}\right), H(X)$ is the generating polynomial of $\left(h_{u}\right)$ and $e(X)$ is the error polynomial. In this case, one might ask how about the $k$-error linear complexity for the complementary sequence $\left(h_{u}^{\prime}\right)$, which in fact is defined by

$$
h_{u}^{\prime}= \begin{cases}1, & \text { if } u \bmod p^{2} \in \cup_{l \in \mathcal{J}} D_{l} \cup P, \quad u \geq 0, \\ 0, & \text { otherwise, }\end{cases}
$$

where $\mathcal{J}$ is a non-empty subset of $\{0,1, \ldots, p-1\}$ with $1 \leq|\mathcal{J}| \leq(p-1) / 2$. (Note that $h_{u}^{\prime}=1$ for $u \in P$, but $h_{u}=0$ in this case.) In particular, we can get some balanced binary sequences when $|\mathcal{J}|=(p-1) / 2$ for certain special applications.

Fortunately, following the same way as the proof of Theorem 1 we get

$$
L C_{k}^{\mathbb{F}_{2}}\left(\left(h_{u}^{\prime}\right)\right)=\left\{\begin{array}{cl}
p^{2}-p+1, & \text { if } 0 \leq k<p-1, \\
p^{2}-p, & \text { if } p-1 \leq k<(p-1)|\mathcal{J}|, \\
p, & \text { if } k=(p-1)|\mathcal{J}| \\
0, & \text { if } k \geq(p-1)|\mathcal{J}|+1,
\end{array}\right.
$$

if $|\mathcal{J}|$ is odd, and otherwise

$$
L C_{k}^{\mathbb{F}_{2}}\left(\left(h_{u}^{\prime}\right)\right)=\left\{\begin{array}{cl}
p^{2}, & \text { if } k=0 \\
p^{2}-p, & \text { if } 1 \leq k<(p-1)|\mathcal{J}|, \\
p, & \text { if } k=(p-1)|\mathcal{J}| \\
0, & \text { if } k \geq(p-1)|\mathcal{J}|+1,
\end{array}\right.
$$

if 2 is a primitive root modulo $p^{2}$.

The statement of the $k$-error linear complexities of $\left(e_{u}\right)$ and $\left(f_{u}\right)$ follows from Theorem 1 directly. We describe it in the following corollary. 
Corollary 1. Let $\left(e_{u}\right)$ and $\left(f_{u}\right)$ be the binary sequences of period $p^{2}$ defined in (5) and (6), respectively. If 2 is a primitive root modulo $p^{2}$, then their $k$-error linear complexity over $\mathbb{F}_{2}$ satisfies

$$
L C_{k}^{\mathbb{F}_{2}}\left(\left(e_{u}\right)\right)=L C_{k}^{\mathbb{F}_{2}}\left(\left(f_{u}\right)\right)=\left\{\begin{array}{cl}
p^{2}-1, & \text { if } k=0, \\
p^{2}-p+1, & \text { if } 1 \leq k<p-1, \\
p^{2}-p, & \text { if } p-1 \leq k<(p-1)^{2} / 2, \\
0, & \text { if } k \geq(p-1)^{2} / 2,
\end{array}\right.
$$

if $p \equiv 3 \bmod 4$, and otherwise

$$
L C_{k}^{\mathbb{F}_{2}}\left(\left(e_{u}\right)\right)=L C_{k}^{\mathbb{F}_{2}}\left(\left(f_{u}\right)\right)=\left\{\begin{array}{cl}
p^{2}-p, & \text { if } 0 \leq k<(p-1)^{2} / 2, \\
0, & \text { if } k \geq(p-1)^{2} / 2 .
\end{array}\right.
$$

For $w=1$, the result is somewhat different because of (4) and we present it in the following separate theorem.

Theorem 2. Let $\left(h_{u}\right)$ be the binary sequence of period $p^{2}$ defined in (10) using polynomial quotients (2) with $w=1$ and a non-empty subset $\mathcal{I}$ of $\{0,1, \ldots, p-1\}$ with $1 \leq|\mathcal{I}| \leq$ $(p-1) / 2$. If 2 is a primitive root modulo $p^{2}$, then the $k$-error linear complexity over $\mathbb{F}_{2}$ of $\left(h_{u}\right)$ satisfies

$$
L C_{k}^{\mathbb{F}_{2}}\left(\left(h_{u}\right)\right)=\left\{\begin{array}{cl}
p^{2}-p+1, & \text { if } 0 \leq k<p \\
p^{2}-p, & \text { if } p \leq k<p|\mathcal{I}|,|\mathcal{I}|>1, \\
0, & \text { if } k \geq p|\mathcal{I}|,
\end{array}\right.
$$

if $|\mathcal{I}|$ is odd, and otherwise

$$
L C_{k}^{\mathbb{F}_{2}}\left(\left(h_{u}\right)\right)=\left\{\begin{array}{cl}
p^{2}-p, & \text { if } 0 \leq k<p|\mathcal{I}|, \\
0, & \text { if } k \geq p|\mathcal{I}|
\end{array}\right.
$$

Proof. The proof is similar to that of Theorem 1. Here we present a sketch. Let

$$
H_{k}(X)=\sum_{l \in \mathcal{I}} D_{l}(X)+\sum_{l \in \mathcal{I}} X^{l p}+e(X) \in \mathbb{F}_{2}[X]
$$

be the generating polynomial of the sequence obtained from $\left(h_{u}\right)$ by changing exactly $k$ terms of $\left(h_{u}\right)$ per period, where $e(X)$ is the corresponding error polynomial with $k$ many monomials.

For $k<p|\mathcal{I}|$, under the assumption of 2 being primitive root modulo $p^{2}$, we can show $H_{k}\left(\beta^{n}\right) \neq 0$ for all $n \in \mathbb{Z}_{p^{2}}^{*}$, as proved in Theorem 1. So we only need to determine the number of roots of the form $\beta^{i p}(0 \leq i<p)$ for $H_{k}(X)$. By Lemma 1, we have

$$
\begin{aligned}
H_{k}\left(\beta^{i p}\right) & =\sum_{l \in \mathcal{I}} D_{l}\left(\beta^{i p}\right)+\sum_{l \in \mathcal{I}}\left(\beta^{i p}\right)^{l p}+e\left(\beta^{i p}\right) \\
& =e\left(\beta^{i p}\right)+ \begin{cases}|\mathcal{I}|, & \text { if } i=0, \\
0, & \text { if } 1 \leq i<p .\end{cases}
\end{aligned}
$$

For odd $|\mathcal{I}|$, all $\beta^{i p}(1 \leq i<p)$ are roots of $H_{k}(X)$ when $k=0$ and $H_{k}(X)$ has one more root if $e(X)$ satisfies

$$
e\left(\beta^{i p}\right)= \begin{cases}1, & \text { if } i=0, \\ 0, & \text { if } 1 \leq i<p,\end{cases}
$$


from which we derive by Lemma 2

$$
e(X) \equiv 1+X+X^{2}+\ldots+X^{p-1} \quad\left(\bmod X^{p}-1\right) .
$$

That is to say, only that $e(X)$ modulo $X^{p}-1$ is of the form above, which contains $p$ terms, can guarantee that all $\beta^{i p}(0 \leq i<p)$ are roots of $H_{k}(X)$, thus

$$
L C_{k}^{\mathbb{F}_{2}}\left(\left(h_{u}\right)\right)=L C_{0}^{\mathbb{F}_{2}}\left(\left(h_{u}\right)\right)=p^{2}-p+1 \text { for } 0 \leq k<p
$$

and

$$
L C_{k}^{\mathbb{F}_{2}}\left(\left(h_{u}\right)\right)=L C_{p}^{\mathbb{F}_{2}}\left(\left(h_{u}\right)\right)=p^{2}-p \text { for } p \leq k<p|\mathcal{I}| .
$$

For even $|\mathcal{I}|$, all $\beta^{i p}(0 \leq i<p)$ are roots of $H_{0}(X)$ and any $e(X)$ with $k$ terms for $k<p|\mathcal{I}|$ will not increase the number of the common roots of $H_{k}(X)$ and $X^{p^{2}}-1$. Then the result follows.

We restrict that 2 is a primitive root modulo $p^{2}$ in the theorems above. A conjecture of Artin suggests that approximately $3 / 8$ of all primes have 2 as a primitive element ([39, p.81]), and it is very seldom that a primitive element modulo the prime $p$ is not a primitive element modulo $p^{2}$. If 2 is not a primitive root modulo $p^{2}$, it seems that our method is not suitable for computing the exact number of the common roots of $H_{k}(X)$ and $X^{p^{2}}-1$ without additional ideas, as mentioned in the proof of Theorem 1 . But we have some partial results, as described in the following theorem, under certain special conditions. We conjecture that Theorems 1 and 2 are true for most primes $p$, e.g. $p$ satisfying $2^{p-1} \not \equiv 1\left(\bmod p^{2}\right)$, see [19, 10] for the applications of such primes. We note that $2^{p-1} \not \equiv 1\left(\bmod p^{2}\right)$ if and only if the order of 2 modulo $p^{2}$ is lager than $p$.

Theorem 3. Let $\mathcal{I} \subseteq\{0,1, \ldots, p-1\}$ with $1 \leq|\mathcal{I}| \leq(p-1) / 2$ and the order of 2 modulo $p^{2}$ be $\lambda p$ with $1<\lambda \leq p-1$ and $\lambda \mid(p-1)$.

(i). Let $\left(h_{u}\right)$ be the binary sequence of period $p^{2}$ defined in (9) using polynomial quotients (2) with $w \geq 2$ and $\mathcal{I}$.

(ii). Let $\left(h_{u}\right)$ be the binary sequence of period $p^{2}$ defined in (10) using polynomial quotients (2) with $w=1$ and $\mathcal{I}$.

If $0 \leq k<(p-1)|\mathcal{I}|$ for (i) or $0 \leq k<p|\mathcal{I}|$ for (ii), the $k$-error linear complexity over $\mathbb{F}_{2}$ of $\left(h_{u}\right)$ satisfies

$$
L C_{k}^{\mathbb{F}_{2}}\left(\left(h_{u}\right)\right) \geq \lambda p
$$

and otherwise $L C_{k}^{\mathbb{F}_{2}}\left(\left(h_{u}\right)\right)=0$.

Proof. First for $0 \leq k<(p-1)|\mathcal{I}|$ for (i), according to the proof of Theorem 1, there do exist an $n_{0} \in \mathbb{Z}_{p^{2}}^{*}$ such that $H_{k}\left(\beta^{n_{0}}\right) \neq 0$ for $H_{k}(X)$, the generating polynomial of the sequence obtained from $\left(h_{u}\right)$ by changing exactly $k$ terms of $\left(h_{u}\right)$ per period. (Otherwise, we will get a more accurate result, as described in Theorem 1.) Thus there are at least $\lambda p$ many $n \in\left\{n_{0} 2^{j} \bmod p^{2}: 0 \leq j<\lambda p\right\}$ such that $H_{k}\left(\beta^{n}\right) \neq 0$. Then the result follows.

For the case of $0 \leq k<p|\mathcal{I}|$ for (ii), the discussion is similar by using the proof of Theorem 2 . 


\section{$3 k$-Error Linear Complexity over $\mathbb{F}_{p}$}

In this section, we view the binary sequences $\left(h_{u}\right)$ defined in $(9)$ and $(10)$ as sequences over $\mathbb{F}_{p}$ and consider their ( $k$-error) linear complexity over $\mathbb{F}_{p}$, which is also an interesting problem for binary sequences. Such kind of work has been done in many references, such as $[2,3,4,15,8,18,28,30,32,33$.

We will employ the $j$-th Hasse derivative of a polynomial $F(x)=a_{0}+a_{1} X+\ldots+$ $a_{T-1} X^{T-1} \in \mathbb{F}_{p}[X]$, which is defined to be

$$
F^{(j)}(X)=\sum_{n=j}^{T-1}\left(\begin{array}{l}
n \\
j
\end{array}\right) a_{i} X^{n-j}, j \geq 1 .
$$

The multiplicity of $\mu$ as a root of $F(X)$ is $n$ if $F(\mu)=F^{(1)}(\mu)=\ldots=F^{(n-1)}(\mu)=0$ and $F^{(n)}(\mu) \neq 0$, see e.g. [34, Ch 6.4] for details.

Before presenting the main results, we introduce a technical lemma, which will be used in the proofs.

Lemma 3. With notations of $D_{l}(0 \leq l<p)$ defined in Section 1. Let $D_{l}(X)=\sum_{u \in D_{l}} X^{u} \in$ $\mathbb{F}_{p}[X]$ and $D_{l}^{(j)}(X)$ be the $j$-th Hasse derivative of $D_{l}(X)$ for $0 \leq l<p$. Then for $0 \leq l<p$, we have

$$
D_{l}(X) \equiv(X-1)^{p-1}-1 \quad\left(\bmod X^{p}-1\right)
$$

and hence

$$
D_{l}(1)=p-1, D_{l}^{(j)}(1)=0 \text { and } D_{l}^{(p-1)}(1)=1,
$$

where $1 \leq j \leq p-2$.

Proof. In the proof of Lemma 1, we have shown that

$$
\left\{u \quad(\bmod p): u \in D_{l}\right\}=\mathbb{Z}_{p}^{*}, 0 \leq l<p,
$$

i.e.,

$$
D_{l}=\left\{v+m_{l v} p: 1 \leq v<p, m_{l v}=\left(w v^{w-1}\right)^{-1}\left(l-q_{p, w}(v)\right) \bmod p\right\},
$$

from which we derive

$$
\begin{aligned}
D_{l}(X) & \equiv X+X^{2}+\ldots+X^{p-1} \\
& \equiv\left(1+X+X^{2}+\ldots+X^{p-1}\right)-1 \\
& \equiv \frac{X^{p}-1}{X-1}-1 \\
& \equiv \frac{(X-1)^{p}}{X-1}-1 \\
& \equiv(X-1)^{p-1}-1 \quad\left(\bmod X^{p}-1\right) .
\end{aligned}
$$

Then write

$$
D_{l}(X)=(X-1)^{p-1}-1+\eta(X)\left(X^{p}-1\right) \in \mathbb{F}_{p}[X]
$$


for some $\eta(X) \in \mathbb{F}_{p}[X]$, it is easy to check the rest equalities by using

$$
D_{l}^{(j)}(X)=\left(\begin{array}{c}
p-1 \\
j
\end{array}\right)(X-1)^{p-1-j}+\sum_{\substack{j_{1}+j_{2}=j \\
0 \leq j_{1}, j_{2} \leq j}}\left(\begin{array}{c}
p \\
j_{1}
\end{array}\right)(X-1)^{p-j_{1}} \eta^{\left(j_{2}\right)}(X)
$$

for $1 \leq j<p$, where we use $X^{p}-1=(X-1)^{p}$.

Now we present our main results.

Theorem 4. Let $\left(h_{u}\right)$ be the (binary) sequence of period $p^{2}$ defined in 10) using polynomial quotients (2) with $w=1$ and a non-empty subset $\mathcal{I}$ of $\{0,1, \ldots, p-1\}$ with $1 \leq|\mathcal{I}| \leq$ $(p-1) / 2$. Then we have

$$
L C_{k}^{\mathbb{F}_{p}}\left(\left(h_{u}\right)\right)=L C_{0}^{\mathbb{F}_{p}}\left(\left(h_{u}\right)\right)=p^{2}-p+1
$$

for $0 \leq k<p$, and $L C_{k}^{\mathbb{F} p}\left(\left(h_{u}\right)\right) \leq p^{2}-p$ for $k \geq p$.

Proof. Let

$$
H_{k}(X)=\sum_{l \in \mathcal{I}}\left(D_{l}(X)+X^{l p}\right)+e(X) \in \mathbb{F}_{p}[X]
$$

be the generating polynomial of the sequence obtained from $\left(h_{u}\right)$ by changing exactly $k$ terms of $\left(h_{u}\right)$ per period, where $e(X)$ is the corresponding error polynomial with $k$ many monomials. In particular, $H_{0}(X)=\sum_{l \in \mathcal{I}}\left(D_{l}(X)+X^{l p}\right)$ is the generating polynomial of $\left(h_{u}\right)$. Since $X^{p^{2}}-1=(X-1)^{p^{2}}$ over $\mathbb{F}_{p}$, we only need to consider the multiplicity of 1 as a root of $H_{k}(X)$.

It is easy to check by Lemma 3 that

$$
H_{0}(1)=0, H_{0}^{(j)}(1)=0 \text { for } 1 \leq j \leq p-2, H_{0}^{(p-1)}(1)=|\mathcal{I}| \neq 0,
$$

where $H_{0}^{(j)}(X)$ is the $j$-th Hasse derivative of $H_{0}(X)$. So we have

$$
(X-1)^{p-1} \| H_{0}(X)
$$

where the notation '\|l' means $(X-1)^{p-1} \mid H_{0}(X)$ but $(X-1)^{p} \nmid H_{0}(X)$. Hence the linear complexity of $\left(h_{u}\right)$ is

$$
L C^{\mathbb{F}_{p}}\left(\left(h_{u}\right)\right)=L C_{0}^{\mathbb{F}_{p}}\left(\left(h_{u}\right)\right)=p^{2}-(p-1)
$$

by (8).

Now we consider the case of $k \geq 1$. For $e(X)$ with $k$ terms, since $(X-1)^{p-1} \| H_{0}(X)$ it is easy to see that

$$
(X-1)^{p-1} \| H_{k}(X)
$$

if $(X-1)^{p} \mid e(X)$, and

$$
(X-1)^{m} \| H_{k}(X)
$$


if $(X-1)^{m} \| e(X)$ and $m \leq p-2$. So for such $e(X)$, the multiplicity of 1 as a root of $H_{k}(X)$ is at most $p-1$ and hence the $k$-error linear complexity will not decrease. Now we assume $(X-1)^{p-1} \| e(X)$ and write

$$
e(X) \equiv(X-1)^{p-1}\left(\alpha_{0} X^{v_{0}}+\alpha_{1} X^{v_{1}}+\ldots+\alpha_{t-1} X^{v_{t-1}}\right) \quad\left(\bmod X^{p}-1\right),
$$

where $\alpha_{0}, \ldots, \alpha_{t-1} \in \mathbb{F}_{p}^{*}, \alpha_{0}+\ldots+\alpha_{t-1} \neq 0$ and $0 \leq v_{0}<v_{1}<\ldots<v_{t-1} \leq p^{2}-p$. Using the facts that

$$
(X-1)^{p-1}=\frac{X^{p}-1}{X-1}=1+X+X^{2}+\ldots+X^{p-1} \in \mathbb{F}_{p}[X]
$$

and

$$
\begin{aligned}
& \left(1+X+X^{2}+\ldots+X^{p-1}\right) \cdot \alpha_{i} X^{v_{i}} \\
\equiv & \left(1+X+X^{2}+\ldots+X^{p-1}\right) \cdot\left(\alpha_{i} X^{v_{i}}-\alpha_{i} X^{v_{i}-1}+\alpha_{i} X^{v_{i}-1}\right) \\
\equiv & \left(1+X+X^{2}+\ldots+X^{p-1}\right) \cdot \alpha_{i} X^{v_{i}-1} \\
\equiv & \ldots \\
\equiv & \left(1+X+X^{2}+\ldots+X^{p-1}\right) \cdot \alpha_{i} \quad\left(\bmod X^{p}-1\right)
\end{aligned}
$$

we get

$$
e(X) \equiv\left(1+X+X^{2}+\ldots+X^{p-1}\right)\left(\alpha_{0}+\alpha_{1}+\ldots+\alpha_{t-1}\right) \quad\left(\bmod X^{p}-1\right) .
$$

That is to say, $e(X)$ modulo $X^{p}-1$ should be of the form above and it has at least $p$ terms if $(X-1)^{p-1} \| e(X)$.

Hence we conclude that, if $1 \leq k<p$, the multiplicity of 1 as a root of $e(X)$, which contains $k$ terms, is not equal to $p-1$. (Otherwise, $e(X)$ modulo $X^{p}-1$ has at most $k$ terms, a contradiction.) So we have

$$
(X-1)^{p} \nmid H_{k}(X) \text { for } 1 \leq k<p
$$

and we derive the desired result.

For $k=p$, one can choose

$$
e(x)=-\alpha\left(1+X+X^{2}+\ldots+X^{p-1}\right)=-\alpha(X-1)^{p-1} \in \mathbb{F}_{p}[X],
$$

where $\alpha=\left.\frac{H_{0}(X)}{(X-1)^{p-1}}\right|_{X=1} \neq 0$. From

$$
H_{k}(X)=H_{0}(X)+e(X)=(X-1)^{p-1}\left(\frac{H_{0}(X)}{(X-1)^{p-1}}-\alpha\right),
$$

we find $(X-1)^{p} \mid H_{k}(X)$ and the value $L C_{p}^{\mathbb{F}_{p}}\left(\left(h_{u}\right)\right) \leq p^{2}-p$.

Theorem 5. Let $\left(h_{u}\right)$ be the (binary) sequence of period $p^{2}$ defined in (9) using polynomial quotients (2) with $2 \leq w \leq p-1$ and a non-empty subset $\mathcal{I}$ of $\{0,1, \ldots, p-1\}$ with $1 \leq|\mathcal{I}| \leq(p-1) / 2$. Then we have

$$
L C_{k}^{\mathbb{F}_{p}}\left(\left(h_{u}\right)\right)=\left\{\begin{array}{cl}
p^{2}, & \text { if } k=0 \\
p^{2}-p+1, & \text { if } 1 \leq k<p-1,
\end{array}\right.
$$

and $L C_{k}^{\mathbb{F}_{p}}\left(\left(h_{u}\right)\right) \leq p^{2}-p$ for $k \geq p-1$. 
Proof. Let

$$
H_{k}(X)=\sum_{l \in \mathcal{I}} D_{l}(X)+e(X) \in \mathbb{F}_{p}[X]
$$

be the generating polynomial of the sequence obtained from $\left(h_{u}\right)$ by changing exactly $k$ terms of $\left(h_{u}\right)$ per period, where $e(X)$ is the corresponding error polynomial with $k$ many monomials.

We check that $H_{0}(1)=(p-1)|\mathcal{I}| \neq 0 \bmod p$, hence $L C^{\mathbb{F}_{p}}\left(\left(h_{u}\right)\right)=p^{2}$ by $(8)$.

Below we consider the case of $k=1$. For $e(X)=\xi X^{i p}$ for any $\xi \in \mathbb{F}_{p}^{*}$ and $0 \leq i<p$, we have

$$
H_{1}(1)=(p-1)|\mathcal{I}|+\xi \begin{cases}=0, & \text { if } \xi=|\mathcal{I}| \\ \neq 0, & \text { otherwise }\end{cases}
$$

and

$$
\begin{aligned}
\left.H_{1}^{(j)}(X)\right|_{X=1} & =\left.\sum_{l \in \mathcal{I}} D_{l}^{(j)}(X)\right|_{X=1}+\left.\left(\begin{array}{c}
i p \\
j
\end{array}\right) \xi X^{i p-j}\right|_{X=1} \\
& = \begin{cases}0, & \text { if } 1 \leq j \leq p-2, \\
|\mathcal{I}|, & \text { if } j=p-1\end{cases}
\end{aligned}
$$

by Lemma 3 , So we derive

$$
(X-1)^{p-1}|| H_{1}(X) \text { if } \xi=|\mathcal{I}|
$$

and

$$
(X-1) \nmid H_{1}(X) \text { if } \xi \neq|\mathcal{I}| .
$$

For $e(X)=\xi X^{n}$ for any $\xi \in \mathbb{F}_{p}^{*}$ and $n \in \mathbb{Z}_{p^{2}}^{*}$, we find that $H_{1}(1)=(p-1)|\mathcal{I}|+\xi$ and

$$
\left.H_{1}^{(1)}(X)\right|_{X=1}=\left.\sum_{l \in \mathcal{I}} D_{l}^{(1)}(X)\right|_{X=1}+\left.n \xi X^{n-1}\right|_{X=1}=n \xi \neq 0
$$

hence the multiplicity of 1 as a root of $H_{1}(X)$ is $\leq 1$. So we conclude that

$$
L C_{1}^{\mathbb{F}_{p}}\left(\left(h_{u}\right)\right)=p^{2}-(p-1) .
$$

Now we want to find the smallest $k \geq 2$ such that

$$
(X-1)^{p} \mid H_{k}(X) .
$$

From $H_{k}(1)=0$ and $H_{k}^{(j)}(1)=0$ for $1 \leq j \leq p-1$, we have

$$
\left\{\begin{array}{l}
e(1)=|\mathcal{I}| \\
e^{(j)}(1)=0 \text { for } 1 \leq j \leq p-2 \\
e^{(p-1)}(1)=(p-1)|\mathcal{I}|
\end{array}\right.
$$

by Lemma 3 , where $e^{(j)}(X)$ is the $j$-th Hasse derivative of $e(X)$. We define a new polynomial $\widetilde{e}(X) \in \mathbb{F}_{p}[X]$ with

$$
\widetilde{e}(X)=e(X)+(p-|\mathcal{I}|) X^{i p}
$$


for some $0 \leq i<p$, and compute

$$
\widetilde{e}(1)=0, \widetilde{e}^{(j)}(1)=0 \text { for } 1 \leq j \leq p-2, \widetilde{e}^{(p-1)}(1)=(p-1)|\mathcal{I}| \neq 0 .
$$

Then we have $(X-1)^{p-1} \| \widetilde{e}(X)$. Following the proof of Theorem 4 , we derive

$$
\widetilde{e}(X) \equiv \mu\left(1+X+X^{2}+\ldots+X^{p-1}\right) \quad\left(\bmod X^{p}-1\right)
$$

for $\mu \in \mathbb{F}_{p}^{*}$. Hence $e(X)$ should be of the form

$$
e(X)=\widetilde{e}(X)+|\mathcal{I}| X^{i p} \equiv|\mathcal{I}|+\mu\left(1+X+X^{2}+\ldots+X^{p-1}\right) \quad\left(\bmod X^{p}-1\right),
$$

which contains at least $p-1$ terms, since $|\mathcal{I}|+\mu$ can take 0 as its output. Hence $k \geq p-1$.

So we conclude that if $1 \leq k<p-1,(X-1)^{p \nmid} H_{k}(X)$, from which the first desired result follows. For $k=p-1$, one can directly choose

$$
e(x)=-|\mathcal{I}|\left(X+X^{2}+\ldots+X^{p-1}\right)=|\mathcal{I}|-|\mathcal{I}|(X-1)^{p-1} \in \mathbb{F}_{p}[X],
$$

and then compute

$$
H_{k}(1)=H_{0}(1)-(p-1)|\mathcal{I}|=0
$$

and

$$
\left.H_{k}^{(j)}(X)\right|_{X=1}=\left.H_{0}^{(j)}(X)\right|_{X=1}-\left.|\mathcal{I}|\left(\begin{array}{c}
p-1 \\
j
\end{array}\right)(X-1)^{p-1-j}\right|_{X=1}=0
$$

for $1 \leq j \leq p-1$ by Lemma 3 , so we have $(X-1)^{p} \mid H_{k}(X)$ and $L C_{p-1}^{\mathbb{F}_{p}}\left(\left(h_{u}\right)\right) \leq p^{2}-p$.

It seems difficult for us to consider the case of larger $k$ without additional ideas. We leave it open. However, motivated by [3, 4], we have a more accurate upper bound for $\mathcal{I}$ being the set of quadratic non-residues modulo $p$. In this case, $\left(h_{u}\right)$ in $(9)$ or $(10)$ is in fact $\left(f_{u}\right)$ defined in (6).

Since

$$
\begin{aligned}
q_{p, w}(u) & \equiv q_{p, w}\left(i_{0}+i_{1} p\right) \\
& \equiv \sum_{c=0}^{p-1} q_{p, w}(c)\left(1-\left(i_{0}-c\right)^{p-1}\right)+w i_{0}^{w-1} i_{1} \quad(\bmod p)
\end{aligned}
$$

for all integers $u \equiv i_{0}+i_{1} p\left(\bmod p^{2}\right)$ with $0 \leq i_{0}, i_{1}<p$, according to [4] we see that $\left(f_{u}\right)$ can be represented by

$$
f_{i_{0}+i_{1} p+j p^{2}}=\rho\left(i_{0}, i_{1}\right) \text { for all integers } 0 \leq i_{0}, i_{1}<p \text { and } j,
$$

where the multivariate polynomial $\rho\left(X_{0}, X_{1}\right) \in \mathbb{F}_{p}\left[X_{0}, X_{1}\right] /\left\langle X_{0}^{p}-X_{0}, X_{1}^{p}-X_{1}\right\rangle$ is of the form

$$
\begin{aligned}
\rho\left(X_{0}, X_{1}\right)= & 2^{-1}\left(\sum_{c=0}^{p-1} q_{p, w}(c)\left(1-\left(X_{0}-c\right)^{p-1}\right)+w X_{0}^{w-1} X_{1}\right)^{p-1} \\
& -2^{-1}\left(\sum_{c=0}^{p-1} q_{p, w}(c)\left(1-\left(X_{0}-c\right)^{p-1}\right)+w X_{0}^{w-1} X_{1}\right)^{\frac{p-1}{2}} .
\end{aligned}
$$


We reduce $\rho\left(X_{0}, X_{1}\right)$ modulo $X_{0}^{p}-X_{0}$ and $X_{1}^{p}-X_{1}$ such that the degree strictly less than $p$ in each indeterminate. And then the linear complexity over $\mathbb{F}_{p}$ of $\left(f_{u}\right)$ equals to $1+\operatorname{deg}\left(\rho\left(X_{0}, X_{1}\right)\right)$, we refer the reader to [6, Theorem 8] for the assertion and the definition of the degree of multivariate polynomials.

For $2 \leq w<p$, Substituting 0 by $2^{-1}\left(=\frac{p+1}{2}\right)$ at those positions $u$ with $u \bmod p^{2} \in D_{0} \cup P$ in $\left(f_{u}\right)$, we get a new sequence $\left(\bar{f}_{u}\right)$ represented by the polynomial

$$
2^{-1}-2^{-1}\left(\sum_{c=0}^{p-1} q_{p, w}(c)\left(1-\left(X_{0}-c\right)^{p-1}\right)+w X_{0}^{w-1} X_{1}\right)^{\frac{p-1}{2}},
$$

from which we derive after some simple calculations

$$
L C^{\mathbb{F}_{p}}\left(\left(\bar{f}_{u}\right)\right)= \begin{cases}(p-1) p / 2+p, & \text { if } w \equiv 1 \bmod 2, \\ (p-1) p / 2+(p-1) / 2+1, & \text { otherwise }\end{cases}
$$

by [6, Theorem 8]. Since $\left|D_{0} \cup P\right|=2 p-1$, we obtain an upper bound on the $k$-error linear complexity of $\left(f_{u}\right)$ defined in $(6)$ as follows

$$
L C_{k}^{\mathbb{F}_{p}}\left(\left(f_{u}\right)\right) \leq \begin{cases}(p-1) p / 2+p, & \text { if } w \equiv 1 \bmod 2, \\ (p-1) p / 2+(p-1) / 2+1, & \text { otherwise }\end{cases}
$$

for $k \geq 2 p-1$.

For $w=1$, We only use $\{0\}$ instead of $P$ above and obtain

$$
L C_{k}^{\mathbb{F}_{p}}\left(\left(f_{u}\right)\right) \leq(p-1) p / 2+1
$$

for $k \geq p$.

Finally, we mention a lower bound on the $k$-error linear complexity over $\mathbb{F}_{p}$ of $\left(h_{u}\right)$ defined in $(9)$ or $(10)$. From [6, Theorem 8], each $p^{2}$-periodic sequence over $\mathbb{F}_{p}$ can be represented by a unique polynomial $\varrho\left(X_{0}, X_{1}\right) \in \mathbb{F}_{p}\left[X_{0}, X_{1}\right] /\left\langle X_{0}^{p}-X_{0}, X_{1}^{p}-X_{1}\right\rangle$ with $\operatorname{deg}_{X_{1}}\left(\varrho\left(X_{0}, X_{1}\right)\right) \geq 1$, otherwise the period is reduced to $p$. We find by (3) that changing at most $k$ (smaller than the weight of $\left(h_{u}\right)$ per-period) terms from $\left(h_{u}\right)$ will not reduce the period, hence the $k$-error linear complexity over $\mathbb{F}_{p}$ is $\geq p+1$.

\section{Concluding Remarks}

In this paper, we study the error linear complexity spectrum (see [27] for details) of $p^{2}$ periodic binary sequences defined from the polynomial quotients, that is, we determine exact values of their $k$-error linear complexity over the finite field $\mathbb{F}_{2}$ for all integers $k$ under the assumption of 2 being a primitive root modulo $p^{2}$. Main results can be described in the following figures, which visually reflect how the linear complexity of the binary sequences decreases as the number $k$ of allowed bit changes increases. It is of interest to consider this problem for the case of 2 being not a primitive root modulo $p^{2}$. We only estimate a lower bound on their $k$-error linear complexity if $2^{p-1} \not \equiv 1\left(\bmod p^{2}\right)$, with which most primes $p$ are satisfied, see [19]. 

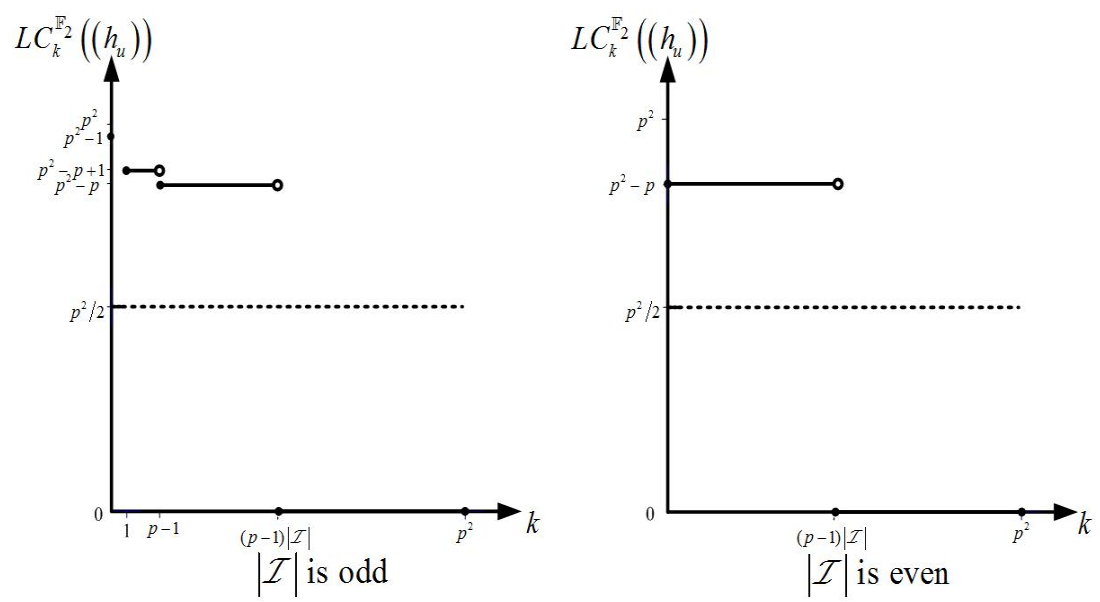

Figure 1: Error linear complexity spectrum of $\left(h_{u}\right)$ when $2 \leq w<p$ (Theorem 1)
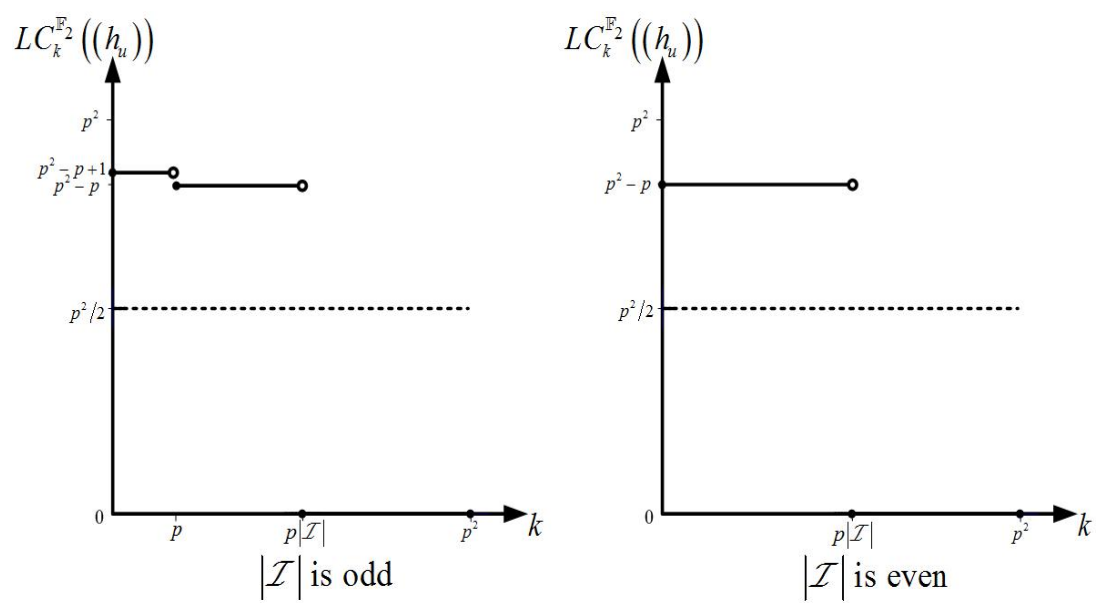

Figure 2: Error linear complexity spectrum of $\left(h_{u}\right)$ when $w=1$ (Theorem 2)

We also view the binary sequences as sequences over the finite field $\mathbb{F}_{p}$ and determine their $k$-error linear complexity over $\mathbb{F}_{p}$ for either $0 \leq k<p$ when $w=1$ or $0 \leq k<p-1$ when $2 \leq w<p$. Results indicate that the linear complexity is large (close to the period) and not significantly reduced by changing a few terms. It is interesting to consider this problem for larger $k$.

We finally remark that, the definition of binary sequences studied in this manuscript is related to generalized cyclotomic classes modulo $p^{2}$, as you can see in Section 1. In particular, the Fermat quotient $q_{p}(-)$ defines a group epimorphism from $\mathbb{Z}_{p^{2}}^{*}$ to $\mathbb{Z}_{p}$ by the fact, see e.g. [37], that

$$
q_{p}(u v) \equiv q_{p}(u)+q_{p}(v) \quad(\bmod p), \operatorname{gcd}(u v, p)=1 .
$$

So if $g$ is a (fixed) primitive root modulo $p^{2}$, we have

$$
D_{0}=\left\{g^{j p} \bmod p^{2}: 0 \leq j<p\right\}
$$


and

$$
D_{l \delta}=g^{j} D_{0}=\left\{g^{j p+l} \bmod p^{2}: 0 \leq j<p\right\}, 1 \leq l<p,
$$

where $\delta=q_{p}(g)$ and the subscript of $D$ is performed modulo $p$. (Note that for $w \neq p-1$, we don't have this property.) Sequences related to cyclotomic classes modulo a prime and generalized cyclotomic classes modulo the product of two distinct primes have been widely investigated since several decades ago, the well-known basic examples are the Legendre sequences and the Jacobi sequences, see [20, 22, 23, 24] and references therein. As we know, the $k$-error linear complexity of the Jacobi sequences and their generalizations [22, 23] has not been solved thoroughly. Hence we hope that our idea and method might be helpful for considering this problem and lead to furtherly study applications of the theory of cyclotomy in cryptography.

\section{Acknowledgements}

The authors wish to thank Arne Winterhof for helpful suggestions.

Z.X.C. was partially supported by the National Natural Science Foundation of China under grant No. 61170246 and the Special Scientific Research Program in Fujian Province Universities of China under grant No. JK2013044.

Z.H.N. was partially supported by the Shanghai Leading Academic Discipline Project under grant No. J50103, the National Natural Science Foundation of China under grants No. 61074135, 61272096 and 61202395, the Program for New Century Excellent Talents in University under grant NCET-12-0620, and the Shanghai Municipal Education Commission Innovation Project.

C.H.W. was partially supported by the Foundation item of the Education Department of Fujian Province of China under grants No. JA12291 and JB12179.

Parts of this paper were written during a very pleasant visit of the first author to RICAM in Linz. He wishes to thank for the hospitality.

\section{References}

[1] T. Agoh, K. Dilcher and L. Skula. Fermat quotients for composite moduli. J. Number Theory 66 (1997) 29-50.

[2] H. Aly and W. Meidl. On the linear complexity and $k$-error linear complexity over $\mathbb{F}_{p}$ of the $d$-ary Sidel'nikov sequence. IEEE Trans. Inform. Theory 53 (2007) 4755-4761.

[3] H. Aly, W. Meidl and A. Winterhof. On the $k$-error linear complexity of cyclotomic sequences. J. Math. Cryptol. 1 (2007) 283-296.

[4] H. Aly and A. Winterhof. On the $k$-error linear complexity over $\mathbb{F}_{p}$ of Legendre and Sidel'nikov sequences. Des. Codes Cryptogr. 40 (2006) 369-374.

[5] H. Aly and A. Winterhof. Boolean functions derived from Fermat quotients. Cryptogr. Commun. 3 (2011) 165-174. 
[6] S. R. Blackburn, T. Etzion, K. G. Paterson. Permutation polynomials, de Bruijn sequences, and linear complexity. J. Combin. Theory Ser. A 76 (1996) 55-82.

[7] J. Bourgain, K. Ford, S. Konyagin and I. E. Shparlinski. On the divisibility of Fermat quotients. Michigan Math. J. 59 (2010) 313-328.

[8] N. Brandstätter and A. Winterhof. $k$-error linear complexity over $\mathbb{F}_{p}$ of subsequences of Sidelnikov sequences of period $\left(p^{r}-1\right) / 3$. J. Math. Cryptol. 3 (2009) 215-225.

[9] M. C. Chang. Short character sums with Fermat quotients. Acta Arith. 152 (2012) $23-38$.

[10] Z. X. Chen and X. N. Du. On the linear complexity of binary threshold sequences derived from Fermat quotients. Des. Codes Cryptogr. 67 (2013) 317-323.

[11] Z. X. Chen and D. Gómez-Pérez. Linear complexity of binary sequences derived from polynomial quotients. Sequences and Their Applications-SETA 2012, 181-189, Lecture Notes in Comput. Sci., 7280, Springer, Berlin, 2012.

[12] Z. X. Chen, L. Hu and X. N. Du. Linear complexity of some binary sequences derived from Fermat quotients. China Commun. 9 (2012) 105-108.

[13] Z. X. Chen, A. Ostafe and A. Winterhof. Structure of pseudorandom numbers derived from Fermat quotients. Arithmetic of Finite Fields-WAIFI 2010, 73-85, Lecture Notes in Comput. Sci., 6087, Springer, Berlin, 2010.

[14] Z. X. Chen and A. Winterhof. Additive character sums of polynomial quotients. Theory and Applications of Finite Fields-Fq10, 67-73, Contemp. Math., 579, Amer. Math. Soc., Providence, RI, 2012.

[15] Z. X. Chen and A. Winterhof. On the distribution of pseudorandom numbers and vectors derived from Euler-Fermat quotients. Int. J. Number Theory 8 (2012) 631-641.

[16] Z. X. Chen and A. Winterhof. Interpolation of Fermat quotients. SIAM J. Discr. Math. 2013 (to appear)

[17] Z. X. Chen. Trace representation and linear complexity of binary sequences derived from Fermat quotients. http://arxiv.org/arXiv:1306.5648, 2013.

[18] J. H. Chung, K. Yang. Bounds on the linear complexity and the 1-error linear complexity over $\mathbb{F}_{p}$ of $M$-ary Sidel'nikov sequences. Sequences and Their Applications-SETA 2006, 74-87, Lecture Notes in Comput. Sci., vol. 4086, Springer, Berlin, 2006.

[19] R. Crandall, K. Dilcher and C. Pomerance. A search for Wieferich and Wilson primes. Math. Comp. 66 (217) (1997) 433-449.

[20] T. W. Cusick, C. S. Ding, A. Renvall. Stream ciphers and number theory. NorthHolland Mathematical Library, 55. North-Holland Publishing Co., Amsterdam, 1998.

[21] C. S. Ding, G. Z. Xiao, W. J. Shan. The stability theory of stream ciphers. Lecture Notes in Computer Science, 561. Springer-Verlag, Berlin, 1991. 
[22] C. S. Ding. Linear complexity of generalized cyclotomic binary sequences of order 2. Finite Fields Appl. 3 (1997) 159-174.

[23] C. S. Ding. Autocorrelation values of generalized cyclotomic sequences of order two. IEEE Trans. Inform. Theory 44 (1998) 1699-1702.

[24] C. S. Ding, T. Helleseth, W. J. Shan. On the linear complexity of Legendre sequences. IEEE Trans. Inform. Theory 44 (1998) 1276-1278.

[25] X. N. Du, Z. X. Chen and L. Hu. Linear complexity of binary sequences derived from Euler quotients with prime-power modulus. Inform. Process. Lett. 112 (2012) 604-609.

[26] X. N. Du, A. Klapper and Z. X. Chen. Linear complexity of pseudorandom sequences generated by Fermat quotients and their generalizations. Inform. Process. Lett. 112 (2012) 233-237.

[27] T. Etzion, N. Kalouptsidis, N. Kolokotronis, K. Limniotis and K. G. Paterson. Properties of the error linear complexity spectrum. IEEE Trans. Inform. Theory 55 (2009) $4681-4686$.

[28] Y. C. Eun, H. Y. Song and G. M. Kyureghyan. One-error linear complexity over $\mathbb{F}_{p}$ of Sidel'nikov sequences. Sequences and Their Applications-SETA 2004, 154-165, Lecture Notes in Comput. Sci., vol. 3486, Springer, Berlin, 2005.

[29] R. Ernvall and T. Metsänkylä. On the $p$-divisibility of Fermat quotients. Math. Comp. 66 (1997) 1353-1365.

[30] M. Z. Garaev, F. Luca, I. E. Shparlinski and A. Winterhof. On the lower bound of the linear complexity over $\mathbb{F}_{p}$ of Sidelnikov sequences. IEEE Trans. Inform. Theory 52 (2006) 3299-3304.

[31] D. Gómez-Pérez and A. Winterhof. Multiplicative character sums of Fermat quotients and pseudorandom sequences. Period. Math. Hungar. 64 (2012) 161-168.

[32] T. Helleseth, S. H. Kim and J. S. No. Linear complexity over $\mathbb{F}_{p}$ and trace representation of Lempel-Cohn-Eastman sequences. IEEE Trans. Inform. Theory 49 (2003) 1548-1552.

[33] T. Helleseth, M. Maas, J. E. Mathiassen and T. Segers. Linear complexity over $\mathbb{F}_{p}$ of Sidel'nikov sequences. IEEE Trans. Inform. Theory 50 (2004) 2468-2472.

[34] R. Lidl and H. Niederreiter. Finite Fields. Second edition. Encyclopedia of Mathematics and its Applications, 20. Cambridge University Press, Cambridge, 1997.

[35] J. L. Massey. Shift register synthesis and BCH decoding. IEEE Trans. Inform. Theory 15 (1969) 122-127.

[36] W. Meidl. How many bits have to be changed to decrease the linear complexity? Des. Codes Cryptogr. 33 (2004) 109-122.

[37] A. Ostafe and I. E. Shparlinski. Pseudorandomness and dynamics of Fermat quotients. SIAM J. Discr. Math. 25 (2011) 50-71. 
[38] M. Sha. The arithmetic of Carmichael quotients. http://arxiv.org/arXiv:1108.2579, 2011.

[39] D Shanks. Solved and Unsolved Problems in Number Theory. Second edition. Chelsea Publishing Company, New York, 1978.

[40] I. D. Shkredov. On Heilbronn's exponential sum. Quart. J. Math. (2012) doi: 10.1093/qmath/has037.

[41] I. E. Shparlinski. Character sums with Fermat quotients. Quart. J. Math. 62 (2011) 1031-1043.

[42] I. E. Shparlinski. Bounds of multiplicative character sums with Fermat quotients of primes. Bull. Aust. Math. Soc. 83 (2011) 456-462.

[43] I. E. Shparlinski. On the value set of Fermat quotients. Proc. Amer. Math. Soc. 140 (2012) 1199-1206.

[44] I. E. Shparlinski. Fermat quotients: Exponential sums, value set and primitive roots. Bull. Lond. Math. Soc. 43 (2011) 1228-1238.

[45] I. E. Shparlinski and A. Winterhof. Distribution of values of polynomial Fermat quotients. Finite Fields Appl. 19 (2013) 93-104.

[46] M. Stamp, C. F. Martin. An algorithm for the $k$-error linear complexity of binary sequences with period $2^{n}$. IEEE Trans. Inform. Theory 39 (1993) 1398-1401. 